\title{
CONVERGENCE OF HEAT KERNELS FOR DEGENERATING HYPERBOLIC SURFACES
}

\author{
LIZHEN JI
}

(Communicated by Dennis A. Hejhal)

\begin{abstract}
For a degenerating family of hyperbolic surfaces $S_{l}(l \geq 0)$, we show that the heat kernel of $S_{l}$ converges to the heat kernel of $S_{0}$. The proof consists of two steps. For small time, we use the Brownian motion interpretation of the heat kernels to prove the convergence. Then we use Gaussian type bounds for the heat kernels and their derivatives and a priori bounds for heat equations to finish the proof.
\end{abstract}

\section{INTRODUCTION}

Let $M_{g} \quad(g \geq 2)$ be the moduli space of Riemann surfaces of genus $g$, and let $\overline{M_{g}}$ be the Deligne-Mumford compactified moduli space of stable Riemann surfaces. For any $S \in \overline{M_{g}}, S$ has a canonical hyperbolic metric. We call such a surface with its hyperbolic metric a hyperbolic surface. There have been many studies devoted to understanding the spectral properties of $S$ near the boundary $\overline{M_{g}} \backslash M_{g}$ (see $[3-6,13]$.

In this note, we study the heat kernel of $S$. Before stating the results, we set up some notation. Let $S_{l} \quad(l \geq 0)$ be a degenerating family of hyperbolic surfaces, i.e., for $l>0, S_{l}$ is compact, while $S_{0}$ is complete, noncompact, and of finite area. The degeneration is caused by the fact that the lengths of several simple, closed geodesics $\gamma_{1}(l), \ldots, \gamma_{m}(l)$ in $S_{l}$ go to zero as $l \rightarrow 0$. These geodesics are referred to as pinching geodesics.

To compare functions on $S_{l}$ and $S_{0}$, we use the globally defined harmonic map of infinite energy $\pi_{l}: S_{0} \rightarrow S_{l}$ constructed by Wolf [12]. The map $\pi_{l}$ is a homeomorphism from $S_{0}$ to $S_{l} \backslash\left\{\gamma_{1}(l), \ldots, \gamma_{m}(l)\right\}$ and intuitively opens up each node of $S_{0}$ (i.e., a pair of cusps) into a pinching geodesic of $S_{l}$. Let $d s_{l}^{2}$ be the hyperbolic metric on $S_{l}$; then $\pi_{l}^{*}\left(d s_{l}^{2}\right)$ converges to $d s_{0}^{2} C^{k}$-uniformly over compact subsets of $S_{0}$.

Let $H_{l}(x, y, t)$ be the heat kernel of $S_{l}$. We then have the following:

Theorem 1.1. For any $(x, y, t) \in S_{0} \times S_{0} \times \mathbb{R}^{+}$,

$$
\lim _{l \rightarrow 0} H_{l}\left(\pi_{l}(x), \pi_{l}(y), t\right)=H_{0}(x, y, t),
$$

and the convergence is uniform over all the compact subsets.

Received by the editors February 22, 1993 and, in revised form, May 21, 1993.

1991 Mathematics Subject Classification. Primary 58G25. 
The above theorem gives the leading term of the asymptotic expansion of $H_{l}\left(\pi_{l}(x), \pi_{l}(y), t\right)$ in $l$ near $l=0$.

A preliminary version of this note was written a few years ago. In the meantime, we received a preprint by J. Jorgenson and R. Lundelius which contains, among other things, a nice proof of a stronger version of the above result $[6$, Theorem 6.1] in the case that $S_{0}$ is connected, based on convergence of the uniformization groups and sphere packing in the upper half plane. Theorem 1.1 also follows from the general theory developed by Hejhal [3]. Our original aim in writing this note was to establish such convergence using only intrinsic arguments, i.e., without using the uniformization theorem, which is only available for surfaces of constant curvature.

The arguments in this note will thus work also for more general types of surfaces with nonconstant curvature. One such example is given by perturbing the metric on $S_{l}$ by a bounded conformal factor so that the curvature is bounded, and $S_{l}$ converges $C^{k}$-uniformly to $S_{0}$ over all compact subsets.

Our proof of Theorem 1.1 will entail two steps.

Step 1. For small $t$, say $t \leq \frac{1}{10}$, the Brownian motion interpretation of heat kernels and the strong Markov property will enable us to cut out negligible long thin collars around the pinching geodesics. We can then directly compare the heat kernels on the remaining compact parts to prove the necessary convergence.

Step 2. For the remaining case, we use upper bounds for the heat kernels and their derivatives and a priori estimates for heat equations to conclude that for any sequence $l_{j} \rightarrow 0$ there is a subsequence $\left\{l_{j}^{\prime}\right\} \subset\left\{l_{j}\right\}$ such that $\lim _{j \rightarrow \infty} H_{l_{j}^{\prime}}\left(\pi_{l_{j}^{\prime}}(x), \pi_{l_{j}^{\prime}}(y), t\right)$ exists. Then by Step 1 and the uniqueness for solutions to the initial problems of heat equations, $\lim _{j \rightarrow \infty} H_{l_{j}^{\prime}}\left(\pi_{l_{j}^{\prime}}(x), \pi_{l_{j}^{\prime}}(y), t\right)=$ $H_{0}(x, y, t)$. Hence, for all $t>0, \lim _{l \rightarrow 0} H_{l}\left(\pi_{l}(x), \pi_{l}(y), t\right)=H_{0}(x, y, t)$.

\section{SeParating the loNG thin COllars awAY From compact Parts}

Let $M$ be a Riemann manifold. A positive function $H(x, y, t) \in$ $C^{\infty}\left(M \times M \times \mathbb{R}^{+}\right)$is called a heat kernel of $M$ if the following conditions are satisfied:

(1) $\frac{\partial}{\partial t} H(x, y, t)+\Delta H(x, y, t)=0$, where $\Delta$ is the Laplace-Beltrami operator of $M$ and normalized to be nonnegative;

(2) $\lim _{t \rightarrow 0} H(x, y, t)=\delta_{y}(x)$, where $\delta_{y}(\cdot)$ is the Dirac delta function at $y ;$ and

(3) $H(x, y, t)=H(y, x, t)$.

If $M$ is complete and its Ricci curvature is bounded from below, then $M$ has a unique heat kernel. If $M$ is compact with nonempty boundary $\partial M$, then we can define its Dirichlet heat kernel with an additional requirement that $H(x, y, t)=0$ if $x \in \partial M$ or $y \in \partial M$. From now on, $M$ is either complete with Ricci curvature bounded from below or compact with boundary.

An important fact concerning the heat kernel is the following Gaussian upper bound due to Li and Yau [8, Corollary 3.1],

Theorem 2.1. Let $M^{n}$ be a complete Riemannian manifold with $\operatorname{Ric}(M) \geq-k$, $k>0$, and $H(x, y, t)$ its unique heat kernel. Then, for each $\delta>0$, there exist 
constants $c(\delta, n), c_{1}(n)$ such that

$$
H(x, y, t) \leq c(\delta, n) V_{x}^{-1 / 2}(\sqrt{t}) V_{y}^{-1 / 2}(\sqrt{t}) \exp \left(-\frac{d^{2}(x, y)}{(4+\delta)^{t}}+c_{1}(n) \delta k t\right),
$$

where $V_{x}(\sqrt{t})$ is the volume of the ball at $x$ of radius $\sqrt{t}$.

The heat kernel $H(x, y, t)$ of $M$ can be interpreted through the Brownian motion on $M$ (see [1, pp. 210-212]). First of all, the Brownian motion on $M$ can be described as follows. Let $M \cup\{\infty\}$ be the one-point compactification of $M$. For any continuous map $\omega:[0, \infty) \rightarrow M \cup\{\infty\}$, let $\xi(\omega)=$ $\inf \{t>0 \mid \omega(t)=\infty\}$. Define

$$
\mathscr{W}=\{\omega:[0, \infty) \rightarrow M \cup\{\infty\} \mid \omega(0) \in M, \omega(t)=\infty, t \geq \xi(\omega)\} ;
$$

i.e., $\mathscr{W}$ consists of those paths which stay at $\infty$ after first reaching there. This is the sample space for the Brownian motion on $M$. Consider cylindrical subsets $A$ of $\mathscr{W}$ :

$$
A=\left\{\omega \in \mathscr{W} \mid\left(\omega\left(t_{1}\right), \omega\left(t_{2}\right), \ldots, \omega\left(t_{n}\right)\right) \in B\right\}
$$

where $0 \leq t_{1}<t_{2}<\cdots<t_{n}$ and $B$ is a Borel set in $\overbrace{M \times M \times \cdots \times M}^{n}$. On the $\sigma$-algebra $\Sigma$ generated by these cylindrical sets, we want to define a probability measure $P_{x}$ for each $x \in M$ such that $P_{x}\left(\mathscr{W}_{x}\right)=1$, where $\mathscr{W}_{x}=$ $\{\omega \in \mathscr{W} \mid \omega(0)=x\}$, and where, for $A \subset \mathscr{W}$ as above,

$$
\begin{aligned}
P_{x}(A)=\int_{B} H\left(x, y_{1}, t_{1}\right) & H\left(y_{1}, y_{2}, t_{2}-t_{1}\right) \\
& \ldots H\left(y_{n-1}, y_{n}, t_{n}-t_{n-1}\right) d V\left(y_{1}, \ldots, y_{n}\right),
\end{aligned}
$$

where $H(x, y, t)$ is the heat kernel of $M$ and $d V\left(y_{1}, \ldots, y_{n}\right)$ is the Riemannian measure on the product $\overbrace{M \times M \times \cdots \times M}^{n}$.

Under the assumption that $\operatorname{Ric}(M) \geq-k>-\infty, \int_{M} H(x, y, t) d V(y)=1$. Thus we can legitimately define $P_{x}(A)$ by the equation above. By the semigroup property of $H(x, y, t), P_{x}$ has a unique extension to the whole $\sigma$ algebra $\Sigma$ and $\left\{\left(P_{x}, \mathscr{W}\right) \mid x \in M\right\}$ is the Brownian motion on $M$.

The Brownian motion is useful in expressing the heat kernel through the following formula: for any Borel set $B \subset M$,

$$
\int_{B} H(x, y, t) d V(y)=P_{x}(\{\omega \in \mathscr{W} \mid \xi(\omega)>t, \omega(t) \in B\}) .
$$

Before proceeding, we introduce the following

Definition. For $B \subset M$, a Borel subset, the first hitting time $T_{B}$ of $B$ is defined by $T_{B}(\omega)=\inf \{t>0 \mid \omega(t) \in B\}$ for any $\omega \in \mathscr{W}$.

The reason we introduce the first hitting time is to express the Dirichlet heat kernel of a regular domain $\Omega \subset M$ in terms of the Brownian motion on $M$. More precisely, let $H_{\Omega}(x, y, t)$ be the Dirichlet heat kernel of $\Omega$ with respect to the induced metric; then, for any $B \subset \Omega$,

(1)

$$
\int_{B} H_{\Omega}(x, y, t) d V(y)=P_{x}\left(\left\{\omega \in \mathscr{W} \mid \omega(t) \in B, t<\min \left\{T_{M \backslash \Omega}(\omega), \xi(\omega)\right\}\right\}\right) .
$$


Now we consider the heat kernels of a degenerating family of surfaces $S_{l}$. For simplicity, we assume that there is only one pinching geodesic $\gamma(l)$ of length $l$. We will also assumie, at first, that it is nonseparating. The long thin collar in $S_{l}$ can be described through the following collar theorem of Keen [7].

Definition. A hyperbolic cylinder with core geodesic of length $l$ and width $2 w$ is a cylinder $\{(r, \theta) \mid-w \leq r \leq w, 0 \leq \theta \leq 1\} /\{(r, 1) \sim(r, 0)\}$ with the metric $d r^{2}+l^{2} \cosh ^{2} r d \theta^{2}$.

Theorem 2.2. There exists a positive constant $\alpha$ such that if $l=|\gamma(l)| \leq \alpha$, the hyperbolic cylinder $C_{l}$ with $\gamma(l)$ as its core geodesic and width $2 \log \left(\frac{1}{l}\right)$ is automatically embedded in $S_{l}$ as a tubular neighborhood of $\gamma(l)$.

For later applications, we denote by $C_{l}(\rho) \quad\left(\rho \leq \log \left(\frac{1}{l}\right)\right)$ the subcollar around $\gamma(l)$ of width $2\left(\log \frac{1}{I}-\rho\right)$. In particular, $C_{l}(0)=C_{l}$.

We are going to cut out $C_{l}(\rho)$ for big $\rho$ and study the influence on the heat kernels. Let $H_{l, \rho}(x, y, t)$ be the Dirichlet heat kernel of $S_{l} \backslash C_{l}(\rho)$.

Proposition 2.3. For any $x, y \in S_{l} \backslash C_{l}\left(\frac{1}{2} \rho\right), 0<t<\frac{1}{10} \rho$, and $l$ with, say, $l e^{\rho}<\frac{1}{2}$, we have

$\left|H_{l}(x, y, t)-H_{l, \rho}(x, y, t)\right| \leq c \max \left\{t^{-1}, t^{-\frac{3}{4}} e^{\frac{1}{2} \rho}, t^{-\frac{1}{2}} e^{\frac{3}{4} \rho}\right\} \exp \left(-\frac{\rho}{10 t}+c_{1} t\right)$,

where $c, c_{1}$ are some absolute positive constants.

Proof. According to the above description, in particular equation (1), for any points $x, y \in S_{l} \backslash C_{l}\left(\frac{1}{2} \rho\right)$ and $\varepsilon>0$ small,

$$
\begin{aligned}
\int_{B(y, \varepsilon)} & H_{l}(x, z, t) d V(z)=P_{x}(\{\omega \mid \omega(t) \in B(y, \varepsilon)\}) \\
= & P_{x}\left(\left\{\omega \mid \omega(t) \in B(y, \varepsilon), T_{C_{l}(\rho)}(\omega)>t\right\}\right) \\
& +P_{x}\left(\left\{\omega \mid \omega(t) \in B(y, \varepsilon), T_{C_{l}(\rho)}(\omega) \leq t\right\}\right) \\
= & \int_{B(y, \varepsilon)} H_{l, \rho}(x, z, t) d V(z)+P_{x}\left(\left\{\omega \mid \omega(t) \in B(y, \varepsilon), T_{C_{l}(\rho)}(\omega) \leq t\right\}\right),
\end{aligned}
$$

where $B(y, \varepsilon)$ is the geodesic ball at $y$ of radius $\varepsilon$. Thus,

$$
\begin{aligned}
& \int_{B(y, \varepsilon)}\left(H_{l}(x, z, t)-H_{l, \rho}(x, z, t)\right) d V(z) \\
& =P_{x}\left(\left\{\omega \mid \omega(t) \in B(y, \varepsilon), \quad T_{C_{l}(\rho)}(\omega) \leq t\right\}\right) .
\end{aligned}
$$

We want to show that the right-hand side of equation (2) is small when $\rho$ is big. This is intuitively clear since it measures the probability of a particle moving into a far away collar (of distance at least $\frac{1}{2} \rho$ ) and coming back in a fixed amount of time. More precisely, by the strong Markov property of Brownian motion (see $[9$, p. 12]),

$$
\begin{aligned}
& P_{x}\left(\left\{\omega \mid \omega(t) \in B(y, \varepsilon), T_{C_{l}(\rho)}(\omega) \leq t\right\}\right) \\
& \quad=\int_{T_{C_{l}(\rho)}(\omega) \leq t} P_{\omega\left(T_{C_{l}(\rho)}(\omega)\right)}\left(\left\{\omega^{\prime} \mid \omega^{\prime}\left(t-T_{C_{l}(\rho)}(\omega)\right) \in B(y, \varepsilon)\right\}\right) d P_{x},
\end{aligned}
$$


where $\omega^{\prime}$ is a new variable in $\mathscr{W}$. Recall

$$
\begin{aligned}
& P_{\omega\left(T_{C_{l}(\rho)}(\omega)\right)}\left(\left\{\omega^{\prime} \mid \omega^{\prime}\left(t-T_{C_{l}(\rho)}(\omega)\right) \in B(y, \varepsilon)\right\}\right) \\
& \quad=\int_{B(y, \varepsilon)} H_{l}\left(\omega\left(T_{C_{l}(\rho)}(\omega)\right), z, t-T_{C_{l}(\rho)}(\omega)\right) d V(z) .
\end{aligned}
$$

By the definition of the first hitting time $T_{C_{l}(\rho)}, \omega\left(T_{C_{l}(\rho)}(\omega) \in \partial\left(C_{l}(\rho)\right)\right.$. Thus for $x, y \in S_{l} \backslash C_{l}\left(\frac{1}{2} \rho\right), d_{l}\left(B(y, \varepsilon), \omega\left(T_{C_{l}(\rho)}(\omega)\right)\right) \geq \frac{1}{2} \rho-\varepsilon$, where $d_{l}(\cdot, \cdot)$ is the distance function of $S_{l}$. We propose to use Theorem 2.1 to show that $H_{l}\left(\omega\left(T_{C_{l}(\rho)}(\omega)\right), z, t-T_{C_{l}(\rho)}(\omega)\right)$ is small when $\rho$ is big.

For fixed $\rho$, the injectivity radius along $\partial C_{l}(\rho)$ is approximately

$$
l \cosh (\tau(l)-\rho) \sim e^{-\rho} \quad \text { as } l \rightarrow 0 .
$$

Thus for $z \in \partial C_{l}(\rho)$, if $\sqrt{t} \geq e^{-\rho}$, then $V_{z}(\sqrt{t}) \geq c \sqrt{t} e^{-\rho}$, and otherwise, $V_{z}(\sqrt{t}) \geq c t$. Hence, when $l e^{\rho} \leq \frac{1}{2}, V_{z}(\sqrt{t}) \geq c \min \left\{t, \sqrt{t} e^{-\rho}\right\}$, where $c$ is some absolute constant. Since $y \in S_{l} \backslash C_{l}(\rho / 2)$, for $z \in B(y, \varepsilon)$, the injectivity radius at $z \geq c^{\prime} e^{-\frac{1}{2} \rho}$, and $V_{z}(\sqrt{t}) \geq c^{\prime} \min \left\{t, \sqrt{t} e^{-\frac{\rho}{2}}\right\}$ for some absolute constant $c^{\prime}>0$. Therefore, by Theorem 2.1 ,

$$
\begin{aligned}
& H_{1}\left(\omega\left(T_{C_{l}(\rho)}(\omega)\right), z, t-T_{C_{l}(\rho)}(\omega)\right) \\
& \quad \leq c(\delta) \max \left\{T^{-1}, T^{-\frac{3}{4}} e^{\frac{1}{2} \rho}, T^{-\frac{1}{2}} e^{\frac{3}{4} \rho}\right\} \exp \left(-\frac{\frac{1}{2} \rho-\varepsilon}{(4+\delta) T}+c_{1} \delta T\right),
\end{aligned}
$$

where $T=t-T_{C_{l}(\rho)(\omega)}$ and $c(\delta)$ is a constant depending only on $\delta$. For $0<$ $\delta \leq 1$, the function on the right-hand side is increasing in $T$ when $T<\frac{1}{10} \rho$; therefore,

$$
\begin{aligned}
& H_{l}\left(\omega\left(T_{C_{l}(\rho)}(\omega)\right), z, t-T_{C_{l}(\rho)}(\omega)\right) \\
& \quad \leq c(\delta) \max \left\{t^{-1}, t^{-\frac{3}{4}} e^{\frac{1}{2} \rho}, t^{-\frac{1}{2}} e^{\frac{3}{4} \rho}\right\} \exp \left(-\frac{\frac{1}{2} \rho-\varepsilon}{(4+\delta) t}+c_{1} \delta t\right),
\end{aligned}
$$

where $t<\frac{1}{10} \rho, 0<\delta \leq 1$.

For simplicity, we take $\delta=1$. Combining equations (2), (3), (4) with the fact that $P_{x}\left(\left\{\omega \in W \mid T_{C_{l}(\rho)}(\omega) \leq t\right\}\right) \leq 1$, we get Proposition 2.3 by letting $\varepsilon \rightarrow 0$.

As $\rho \rightarrow \infty$, the above proposition is interesting only when $\frac{1}{10 t}>\frac{3}{4}$. For $t \leq \frac{1}{10}$, this inequality is obviously satisfied. This is the reason why $\frac{1}{10}$ appears in Step 1 of the proof of Theorem 1.1. Of course, any other small number will also serve the purpose.

For a later application, denote the Dirichlet heat kernel of $S_{l} \backslash \pi_{l}^{-1}\left(\bigcup C_{0}(\rho)\right)$ by $H_{l, \rho}^{*}(x, y, t)$. By similar reasoning, we get the following estimate:

Proposition 2.4. For any $x, y \in S_{l} \backslash \pi_{l}^{-1}\left(C_{0}\left(\frac{1}{2} \rho\right)\right), 0<t<\frac{1}{10} \rho, l e^{\rho} \leq \frac{1}{2}$, $\left|H_{l}(x, y, t)-H_{l, \rho}^{*}(x, y, t)\right| \leq c \max \left\{t^{-1}, t^{-\frac{3}{4}} e^{\frac{1}{2} \rho}, t^{-\frac{1}{2}} e^{\frac{3}{4} \rho}\right\} \exp \left(-\frac{\rho}{10 t}+c_{1} t\right)$, where $c, c_{1}$ are some absolute constants.

Now we formulate similar results for $S_{0}$. The following cusp $C_{0}=\{(r, \theta) \mid$ $r \geq 0,0 \leq \theta \leq 1\} /\{(r, 1) \sim(r, 0)\}$ with metric $d r^{2}+e^{-2 r} d \theta^{2}$ is embedded in $S_{0}$ near each cusp of $S_{0}$. 
Let $C_{0}(\rho) \subset C_{0}$ be the subcusp of $C_{0}$ defined by $C_{0}(\rho)=\left\{(r, \theta) \in C_{0} \mid r \geq\right.$ $\rho\}$ and $S_{0} \backslash \cup C_{0}(\rho)$ the complement of all cusps $C_{0}(\rho)$ inside $S_{0}$ (since $S_{l}$ is assumed to have one pinching geodesic, $S_{0}$ has two cusps). Let $H_{0, \rho}(x, y, t)$ be the Dirichlet heat kernel of $S_{0} \backslash \cup C_{0}(\rho)$. Then by the same argument as above, we get the following

Proposition 2.5. For $x, y \in S_{0} \backslash \bigcup C_{0}\left(\frac{1}{2} \rho\right), 0<t<\frac{1}{10} \rho$,

$\left|H_{0}(x, y, t)-H_{0, \rho}(x, y, t)\right| \leq c \max \left\{t^{-1}, t^{-\frac{3}{4}} e^{\frac{1}{2} \rho}, t^{-\frac{1}{2}} e^{\frac{3}{4} \rho}\right\} \exp \left(-\frac{\rho}{10 t}+c_{1} t\right)$,

where $c, c_{1}$ are some absolute constants.

\section{Proof of Theorem 1.1}

Step 1: $t \leq \frac{1}{10}$. Let $K_{1} \times K_{2} \times K \subset S_{0} \times S_{0} \times\left(0, \frac{1}{10}\right]$ be a compact subset. We need to show that for any $\varepsilon>0$, there exists $\delta>0$ such that for $0<l<\delta$, $(x, y, t) \in K_{1} \times K_{2} \times K$,

$$
\left|H_{l}\left(\pi_{l}(x), \pi_{l}(y), t\right)-H_{0}(x, y, t)\right|<\varepsilon .
$$

By Propositions 2.4 and 2.5, there exist (big) $\rho_{0}>0$ and $\delta_{1}>0$ so that for $0<l<\delta_{1},(x, y, t) \in K_{1} \times K_{2} \times K$,

$$
\begin{gathered}
\left|H_{l}\left(\pi_{l}(x), \pi_{l}(y), t\right)-H_{l, \rho_{0}}^{*}\left(\pi_{l}(x), \pi_{l}(y), t\right)\right|<\frac{1}{4} \varepsilon, \\
\left|H_{0}(x, y, t)-H_{0, \rho_{0}}(x, y, t)\right|<\frac{1}{4} \varepsilon,
\end{gathered}
$$

and hence,

$$
\begin{aligned}
& \left|H_{l}\left(\pi_{l}(x), \pi_{l}(y), t\right)-H_{0}(x, y, t)\right| \\
& \quad<\left|H_{l, \rho_{0}}^{*}\left(\pi_{l}(x), \pi_{l}(y), t\right)-H_{0, \rho_{0}}(x, y, t)\right|+\frac{1}{2} \varepsilon .
\end{aligned}
$$

But $\pi_{l}^{*}\left(d s_{l}^{2}\right)$ converges to $d s_{0}^{2} \quad C^{k}$-uniformly over compact subsets of $S_{0}$. By a theorem of Ray and Singer [10, Proposition 6.1] on variation of heat kernels, for fixed $\rho_{0}$, there exists $0<\delta\left(<\delta_{1}\right)$ such that for $0<l<\delta$, $(x, y, t) \in K_{1} \times K_{2} \times K$,

$$
\left|H_{l, \rho_{0}}^{*}\left(\pi_{l}(x), \pi_{l}(y), t\right)-H_{0, \rho_{0}}(x, y, t)\right|<\frac{1}{2} \varepsilon .
$$

Therefore,

$$
\left|H_{l}\left(\pi_{l}(x), \pi_{l}(y), t\right)-H_{0}(x, y, t)\right|<\frac{1}{2} \varepsilon+\frac{1}{2} \varepsilon=\varepsilon
$$

as required.

Step 2: $t \geq \frac{1}{10}$. To prove Theorem 1.1 for $t \geq \frac{1}{10}$, we need the following estimates on derivatives of heat kernels [11, p. 262].

Theorem 3.1. Let $M^{n}$ be a complete Riemannian manifold with $\operatorname{Ric}(M) \geq$ $-k(k \geq 0)$. Then for all $i \in \mathbb{N}$ and $t_{0}>0$, there exists a constant $C=$ 
$C\left(n, k, t_{0}, i\right)$ such that for $x, y \in M, 0<t \leq t_{0}$,

$$
\begin{aligned}
\left|\frac{\partial^{i}}{\partial t^{i}} H(x, y, t)\right| \leq & C t^{-\frac{1}{2}}\left(1+\frac{d^{2}(x, y)}{t}\right)^{C} \\
& \times(H(x, x, t) H(y, y, t))^{\frac{1}{2}} \exp \left(-\frac{d^{2}(x, y)}{4 t}\right), \\
|\nabla H(x, y, t)| \leq & C t^{-\frac{1}{2}}\left(1+\frac{d^{2}(x, y)}{t}\right)^{C} \\
& \times(H(x, x, t) H(y, y, t))^{\frac{1}{2}} \exp \left(-\frac{d^{2}(x, y)}{4 t}\right),
\end{aligned}
$$

where $d(x, y)$ is the distance function of $M$.

Combining Theorem 2.1 and the fact that $\pi_{l}^{*}\left(d s_{l}^{2}\right)$ converges $C^{k}$-uniformly over compact subsets to $d s_{0}^{2}$, we get the following bounds.

Corollary 3.2. For any compact subset $K_{1} \times K_{2} \times K \subset S_{0} \times S_{0} \times \mathbb{R}^{+}$and any $i \in \mathbb{N}$, there exists a constant $c=c\left(i, K_{1}, K_{2}, K, l_{0}\right)$ such that for $(x, y, t) \in$ $K_{1} \times K_{2} \times K, l \leq l_{0}$,

$$
\begin{aligned}
\left|\frac{\partial^{i}}{\partial t^{i}} H_{l}\left(\pi_{l}(z), \pi_{l}(y), t\right)\right| & \leq c, \\
\left|\nabla_{0} H_{l}\left(\pi_{l}(x), \pi_{l}(y), t\right)\right| & \leq c .
\end{aligned}
$$

As explained in the introduction, we now want to use a normal family argument. We thus need bounds on all (mixed) derivatives of $H_{l}\left(\pi_{l}(x), \pi_{l}(y), t\right)$.

Lemma 3.3. For any $i, k \in \mathbb{N}$ and $K_{1} \times K_{2} \times K$ as above, there exists $c=$ $c\left(i, k, K_{1}, K_{2}, K, l_{0}\right)$ such that for $(x, y, t) \in K_{1} \times K_{2} \times K, l \leq l_{0}$,

$$
\left|\frac{\partial^{i}}{\partial t^{i}} \nabla_{0}^{k} H_{l}\left(\pi_{l}(z), \pi_{l}(y), t\right)\right| \leq c .
$$

Proof. Since $\left(\frac{\partial}{\partial t}+\Delta_{l}\right) H_{l}(x, y, t)=0$,

$$
\left(\frac{\partial}{\partial t}+\Delta_{l}\right) \frac{\partial^{i}}{\partial t^{i}} H_{l}(x, y, t)=0 .
$$

For $x \in K_{1}, \Delta_{l}$ is uniformly elliptic for $l \leq l_{0}$. Then the bound in the lemma follows from a priori estimates for heat equations [2, Theorem 5 in $\S 3.2]$.

We can now finish the proof of Theorem 1.1. From the bound in Lemma 3.3, it follows [2, Theorem 3 in $\S 3.2]$ that for any sequence $l_{j} \rightarrow 0$, there is a subsequence $\left\{l_{j}^{\prime}\right\} \subset\left\{l_{j}\right\}$ such that $H_{l_{j}^{\prime}}\left(\pi_{l_{j}^{\prime}}(x), \pi_{l_{j}^{\prime}}(y), t\right)$ converges $C^{k}$ uniformly over compact subsets to a function $H_{\infty}(x, y, t)$ on $S_{0} \times S_{0} \times \mathbb{R}^{+}$. Of course,

$$
\left(\frac{\partial}{\partial t}+\Delta_{0}\right) H_{\infty}(x, y, t)=0 .
$$

By Step 1 , for $0<t \leq \frac{1}{10}, \lim _{l \rightarrow 0} H_{l}(x, y, t)=H_{0}(x, y, t)$, and hence, $H_{\infty}(x, y, t)=H_{0}(x, y, t)$. Then by the uniqueness of solutions to heat equations, we have $H_{\infty}(x, y, t)=H_{0}(x, y, t)$, i.e., for $t>0$,

$$
\lim _{l_{j}^{\prime} \rightarrow 0} H_{l_{j}^{\prime}}\left(\pi_{l_{j}^{\prime}}(x), \pi_{l_{j}^{\prime}}(y), t\right)=H_{0}(x, y, t) .
$$


By the arbitrary choice of $l_{j} \rightarrow 0$, we get that for any $t>0$,

$$
\lim _{l \rightarrow 0} H_{l}\left(\pi_{l}(x), \pi_{l}(y), t\right)=H_{0}(x, y, t) .
$$

We have thus proved Theorem 1.1 when the pinching geodesic $\gamma(l)$ is nonseparating.

In the case where $\gamma(l)$ is a separating geodesic on $S_{l}$, we need to modify several of the constructs used above. On a disconnected Riemannian manifold $M$, for instance, the heat kernel $H(x, y, t)$ is understood to be 0 whenever $x$ and $y$ belong to disjoint components. Similarly, in this case, we understand that $d(x, y)=+\infty$. After making some minor changes along these lines, the preceding proof is seen to carry over to the separating case as well. The case of several pinching geodesics is handled in much the same way.

\section{ACKNOWLEDGMENT}

I am grateful to the referee for carefully reading this paper and for his advice. He pointed out, among other things, the necessity of emphasizing the case of a separating pinching geodesic $\gamma(l)$ (see the last paragraph of this paper).

\section{REFERENCES}

1. I. Chavel, Eigenvalues in Riemannian geometry, Academic Press, New York, 1984.

2. A. Friedman, Partial differential equations of parabolic type, Prentice-Hall, Englewood Cliffs, NJ, 1964.

3. D. Hejhal, Regular b-groups, degenerating Riemann surfaces and spectral theory, Mem. Amer. Math. Soc., vol. 88, Amer. Math. Soc., Providence, RI, 1990.

4. L. Ji, Spectral degeneration for hyperbolic Riemann surfaces, J. Differential Geom. 38 (1993), 263-313.

5. $\ldots$ The asymptotic behavior of Green's functions for degenerating hyperbolic Riemann surfaces, Math. Z. 212 (1993), 375-394.

6. J. Jorgenson and R. Lundelius, Convergence theorems for relative spectral functions on hyperbolic Riemann surfaces of finite volume, preprint, 1992.

7. L. Keen, Collars on Riemann surfaces, Discontinuous Groups and Riemann Surfaces, Ann. of Math. Stud., no. 79, Princeton Univ. Press, Princeton, NJ, 1974, pp. 263-368.

8. P. Li and S. T. Yau, On the parabolic kernel of the Schrödinger operator, Acta Math. 156 (1986), 153-201.

9. S. C. Port and C. J. Stone, Brownian motions and classical potential theory, Academic Press, New York, 1978.

10. D. Ray and I. M. Singer, R-torsion and the Laplacian on Riemannian manifolds, Adv. Math. 7 (1971), 145-210.

11. N. Th. Varopoulos, Small time Gaussian estimates of heat diffusion kernels, Part 1: The semigroup technique, Bull. Sci. Math. (2) 113 (1989), 253-277.

12. M. Wolf, Infinite energy harmonic maps and degeneration of hyperbolic surfaces, J. Differential Geom. 33 (1991), 487-539.

13. S. Wolpert, Spectral limits for hyperbolic surfaces. I, II, Invent. Math. 108 (1992), 67-89, 91-129.

Department of Mathematics, Massachusetts institute of Technology, Cambridge, MASSACHUSETTS 02139

E-mail address: ji@math.mit.edu 